\title{
Microtubule-Based Transport and the Distribution, Tethering, and Organization of Organelles
}

\author{
Kari Barlan ${ }^{1}$ and Vladimir I. Gelfand ${ }^{2}$ \\ ${ }^{1}$ Department of Molecular Genetics and Cell Biology, University of Chicago, Chicago, Illinois 60637 \\ ${ }^{2}$ Department of Cell and Molecular Biology, Feinberg School of Medicine, Northwestern University, Chicago, \\ Illinois 60611 \\ Correspondence: vgelfand@northwestern.edu
}

\section{SUMMARY}

Microtubules provide long tracks along which a broad range of organelles and vesicles are transported by kinesin and dynein motors. Motor protein complexes also tether cargoes to cytoskeletal filaments, helping facilitate their interaction and communication. The generation of biochemically distinct microtubule subpopulations allows subsets of motors to recognize a given microtubule identity, allowing further organization within the cytoplasm. Both transport and tethering are spatiotemporally regulated through multiple modes, including acute modification of both motor-cargo and motor-track associations by various physiological signals. Strict regulation of intracellular transport is particularly important in specialized cell types such as neurons. Here, we review general mechanisms by which cargo transport is controlled and also highlight examples of transport regulated by multiple mechanisms.

\section{Outline}

1 Introduction

2 Fundamentals of microtubule-based transport

3 Examples of regulated transport

\section{Conclusion}

References 
K. Barlan and V.I. Gelfand

\section{INTRODUCTION}

The ability of a cell to respond and adapt to changing physiological cues relies on continual reorganization of the contents of its cytoplasm. This is accomplished primarily through active transport along cytoskeletal filaments by molecular motor proteins. The collection of cargoes present in any given cell is vast and extremely varied — diverse mem- brane organelles, messenger RNA (mRNA) transcripts, protein complexes, and viruses, among others. In comparison with this diversity, only a small number of components make up the intracellular transport machinery-three families of motors and two types of tracks (Fig. 1). Remarkably, this handful of building blocks can be combined and adapted to create a myriad of finely tuned machines capable of transporting a full range of cellular cargoes.
A

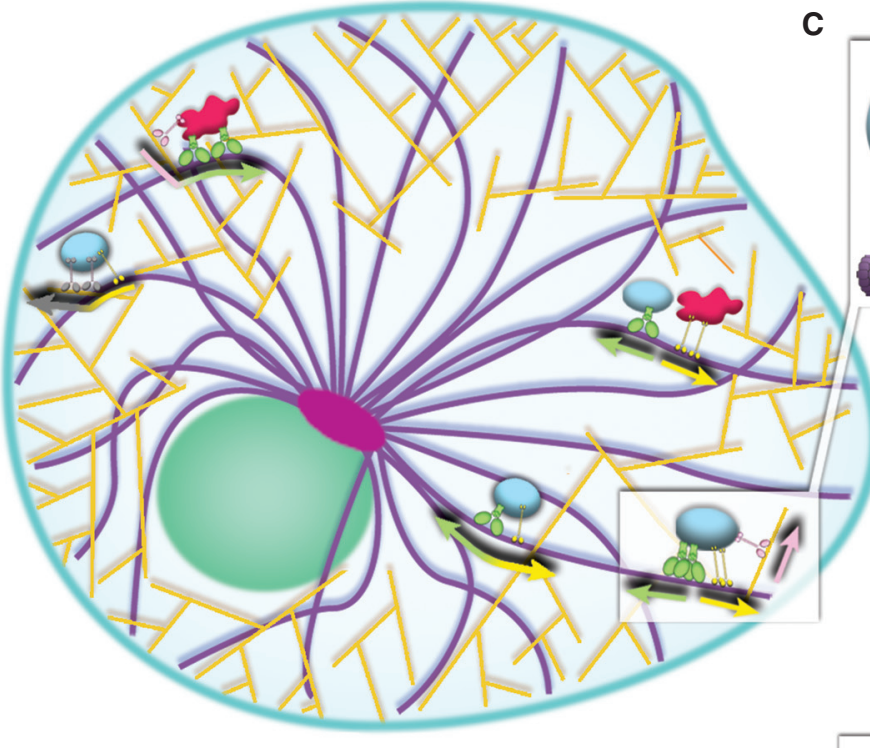

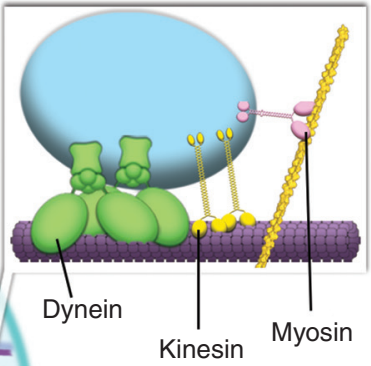

Growth cone

B

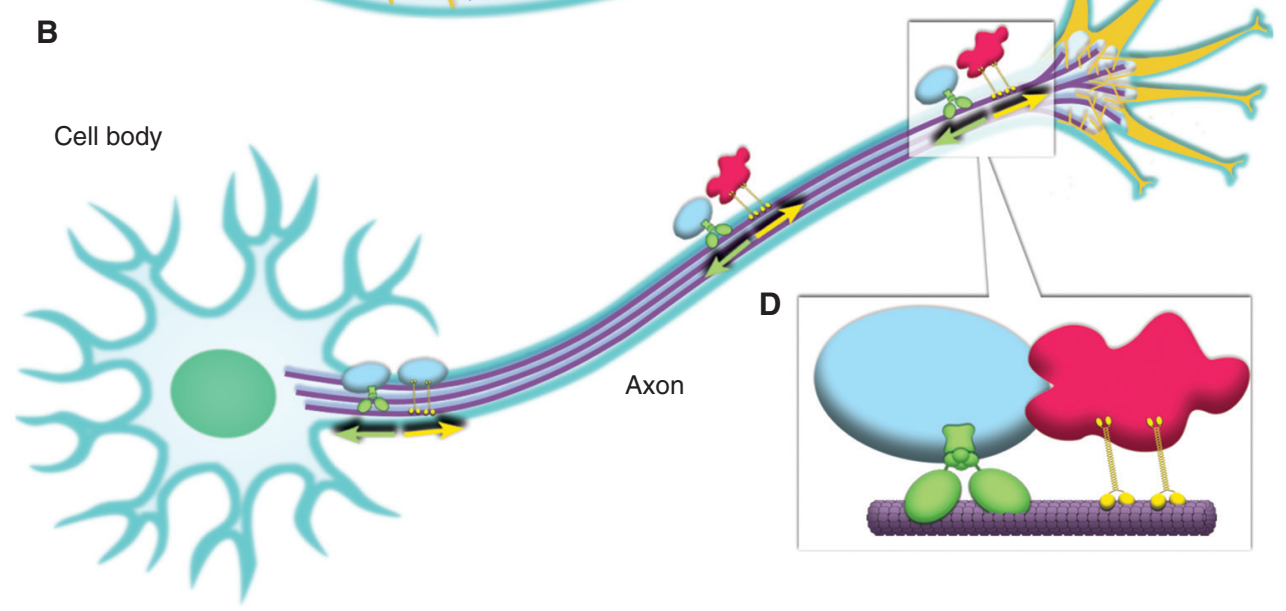

Figure 1. Membrane organelles require multiple motors and cytoskeletal filaments for their distribution. $(A)$ In a steady-state eukaryotic cell, the molecular motors kinesin and dynein transport cargo over long distances along radially arranged microtubules (purple). Actin filaments (dark yellow) are denser near the cell periphery and primarily support short-range transport events by myosin motors. $(B)$ The architecture of the cytoskeletal transport machinery in a neuron is somewhat analogous, with bundles of microtubules (purple tracks) extending from the cell body into the axon and dendrites (not highlighted in this figure), and with actin concentrated in the growth cone at the axon terminal. $(C)$ Organelles are often moved by multiple motors, including motors of opposite polarity and on different cytoskeletal tracks. $(D)$ Microtubules and actin also provide scaffolding where organelle interactions can take place, as attachment to a filament restricts three-dimensional diffusion of organelles to movement in one dimension. The activity of these motors, and the way in which individual cargoes are transported, is likely regulated by molecular factors specific to individual organelles to allow for rapid changes in distribution and motility. (Modified from Barlan et al. 2013b.) Note that the various motors, cargoes, and cellular constituents are not illustrated here to scale. 
Molecular motors have two important, interdependent functions in intracellular transport. Their primary function is to deliver cargoes to discrete cellular locations in response to various physiological stimuli. But motors also play a direct role in facilitating molecular exchanges and chemical interactions between membrane organelles. By tethering organelles to a cytoskeletal track, motors act to limit three-dimensional diffusion to movement in one dimension and thus influence when and where particular intermolecular associations occur and increase the efficiency of component exchange between individual cellular compartments.

Here, we focus on the various ways in which the microtubule cytoskeleton supports the delivery and distribution of cargoes and the types of physiological stimuli that control these events. Examples highlighting the acute regulation of transport in space and time will also be discussed.

\section{FUNDAMENTALS OF MICROTUBULE-BASED TRANSPORT}

\subsection{Motor Proteins Power Cargo Transport along Cytoskeletal Filaments}

Microtubules and actin filaments together make up the tracks along which cargoes can be transported, delivered, and anchored (Fig. 1). As such, they are key components crucial to organizing the rest of the cytoplasm in a cell. The arrangement of each filament network is best suited for a distinct kind of transport. These networks complement one another to provide the architecture necessary to sustain both directed transport events and organelle tethering.

Microtubules are long polymers that are polarized both in their intrinsic structure and also, usually, in their arrangement in the cell. Typically their fast-growing plus ends extend toward the cell periphery, whereas their minus ends are located closer to the cell center and are often anchored at the centrosome, near the nucleus. This wellorganized, radial arrangement of filaments provides tracks for fast transport of cargoes by members of two classes of molecular motor proteins (see Sweeney and Holzbaur 2016). Dynein motors move toward the minus ends of microtubules, whereas most kinesins move toward the plus ends. In contrast to microtubules, actin filaments are shorter and, although the filaments themselves are polarized, they typically form a more randomly oriented meshwork that is most dense near the cell cortex (see Svitkina 2016). Myosin motors move along actin filaments and primarily contribute to localized, short-range movements of cargoes.

Nowhere is the regulation of cargo delivery and distribution more important than in the polarized axon of the neuron. A diverse array of cargoes required for neuronal function and synaptic activity must be transported along microtubules from the cell body, where the cargoes are synthesized, toward axonal synapses that can be many centimeters away. The cytoskeletal architecture of the neuron is analogous to that of other cells (Fig. 1). Bundled microtubules extend along the axon shaft, with their plus ends toward the axon terminal, and actin filaments are enriched in the growth cone, a structure near the distal tip of the axon, and at the synapses.

Understanding how molecular motors function in axonal transport is key to our understanding of a range of neurological diseases (Chevalier-Larsen and Holzbaur 2006; Hirokawa et al. 2010). Mutations in the most crucial components of axonal transport are typically not found in diseases as they are generally lethal. However, various models of neurological disease have lent themselves to the study of axonal transport regulation and have led to the discovery of important accessory proteins (Bowman et al. 2000; Smith et al. 2000; Stowers et al. 2002; Gindhart et al. 2003). Because of the high physiological significance and unique polarized architecture, the neuron has become a popular and powerful model for investigating microtubule-based transport.

Another model system used to study cargo transport comes from the color-changing cells of amphibians and fish. These animals use the regulated transport of pigment-containing organelles, called melanosomes, as a means for rapid color change. This behavior is often induced by changes in the animal's environment. Although melanosomes constantly undergo indiscriminate shortrange movements, their motility becomes highly persistent and synchronous in response to hormonal signals. This results in a dramatic reorganization of pigment within each individual cell and the lightening or darkening of the animal's skin or scales. Details of the regulation of melanosome transport will be discussed below.

The cytoskeletal transport system can also be seized by viruses to aid in their replication and spread. Vaccinia virus, for example, replicates its genome and assembles viral particles in the cytoplasm of cells, and then it uses kinesin-dependent transport to shuttle these viral particles to the plasma membrane for release from the cell (Leite and Way 2015). The timing of viral particle association with kinesin is regulated, in part, by the timing of viral gene expression to ensure that only mature viral particles are transported to the cell periphery. We will discuss an example of viral particle transport below.

Each episode of cargo transport requires three distinct processes: an interaction between motor and cargo, the binding of the motor to a track, and the subsequent movement of the motor along the track. Each one of these processes is subject to a variety of regulatory mechanisms. 


\subsection{Modification of Adaptor Proteins Enables Cargo-Specific Regulation of Transport}

Many motors use autoregulatory mechanisms—so-called autoinhibition - to turn off their catalytic activity when they are not involved in transporting cargo (Sellers et al. 2008; Verhey et al. 2011). Typically, autoinhibition is conferred through intramolecular interactions, which prevent the expensive hydrolysis of ATP and the jamming of cytoskeletal tracks by a large pool of motors not carrying cargo. Binding to cargo is thought to be a key event relieving autoinhibition, leading to microtubule binding and the stimulation of the transport activity of both kinesins and dyneins (Verhey and Hammond 2009; McKenney et al. 2014). Although some cargoes can bind directly to motors, many require adaptor molecules to mediate these interactions (Akhmanova and Hammer 2010). Because a single class of kinesin, for example, contributes to the motility of many dozens of distinct cargoes, regulation of specific motor-cargo interactions provides an efficient way to acutely influence the transport of a single cargo type (Fu and Holzbaur 2014).

Many motor-cargo interactions are regulated by the switch-like behavior of GTPases. When these enzymes cycle between GTP-bound and GDP-bound states, they often change structural conformations, which results in statespecific association with motors or organelles. Dynein adaptor proteins Bicaudal-D1 and Bicaudal-D2 mediate the association of dynein with Golgi membranes through specific interaction with the GTP-bound form of the small GTPase Rab6 (Matanis et al. 2002). Another dynein adaptor, Golgin160, requires GTP-bound Arf1 for Golgi localization. Although Golgin160 links dynein to the Golgi during interphase, its dissociation from membranes during mitosis prevents dynein-mediated membrane retention near the microtubule-organizing center and allows dispersal of Golgi membranes. Cell-cycle regulation of this motor-adaptor interaction, possibly through Arf1 GTP hydrolysis, therefore ensures proper segregation of Golgi membranes between daughter cells following cell division (Yadav et al. 2012). Site-specific phosphorylation and $\mathrm{Ca}^{2+}$ binding can also influence the interaction of an adaptor with motor proteins, microtubules, or cargo. Below we discuss examples of such adaptor modifications that influence cargo motility, including that of mitochondria and viruses.

\subsection{Subpopulations of Microtubules Create Specific Compartments for Organelle Transport and Interaction}

Although all microtubules are polymers comprising $\alpha \beta$ tubulin dimers, not all microtubules are functionally identical. Multiple isoforms of both $\alpha$ and $\beta$ tubulin subunits can combine to form a variety of molecularly distinct microtubules. In vitro experiments show that differences in tubulin isoforms found in the microtubule polymer can influence motor binding, motor velocity, and the overall run length (Sirajuddin et al. 2014). Although the underlying mechanisms are not well understood, multiple congenital neurological disorders have been linked to mutations in specific tubulin isoforms (Tischfield and Engle 2010), suggesting that isoform specificity has physiological consequences as well. In addition to isoform differences, microtubules can be made biochemically distinct through a number of posttranslational modifications and the binding of various microtubule-associated proteins (MAPs) (see Goodson and Jonasson 2016). These accessory changes can create a variety of microtubule subpopulations that serve specific roles in the cell.

Numerous posttranslational modifications can be made to the $\alpha \beta$-tubulin heterodimer in the microtubule polymer, including detyrosination, acetylation, phosphorylation, palmitoylation, polyglutamylation, and polyglycylation of the carboxy-terminal tail of both $\alpha$ and $\beta$ tubulin subunits (Westermann and Weber 2003; Janke 2014). Of these, detyrosination and acetylation have been consistently shown to affect the binding and motility of the kinesin-1 motor, specifically (Liao and Gundersen 1998; Reed et al. 2006; Cai et al. 2009; Konishi and Setou 2009; Hammond et al. 2010). Although kinesin-1 prefers to bind and move along microtubules marked with these particular posttranslational modifications, neither kinesin-2 nor kinesin-3 family members have been found to show a preference for modified microtubules (Cai et al. 2009). The acetylation modification is, curiously, predicted to be on an amino acid residue located in the lumen of the microtubule (Szyk et al. 2014). How a lumenal modification translates to an effect on the binding and motility of motor proteins, which bind to the microtubule surface, is still unknown. Most likely, lumenal acetylation results in a subtle change of the microtubule surface that affects kinesin-1 function. It should be noted that it is not strictly known whether the posttranslational modifications of tubulin described above directly affect interactions of microtubules with motors. It could be that other properties of microtubules, such as whether tubulin dimers are bound to GTP or GDP, affect microtubule conformation (Morikawa et al. 2015), which in turn has an effect on both posttranslational modifications of tubulin and microtubule interactions with motor proteins.

The differences in motor recruitment to microtubules conferred by posttranslational modifications compartmentalize various cellular processes. Mitochondrial division sites are determined, in part, through contact with endoplasmic reticulum (ER) (Friedman et al. 2011). Both 
mitochondria and ER are transported by kinesin-1; thus, acetylated microtubules are the preferred track for ER-mitochondria contacts (Barlan and Gelfand 2010; Friedman et al. 2010).

The preference of kinesin- 1 for modified microtubules is also important for axon specification in developing neurons, which extend multiple microtubule-rich processes (called neurites) from the cell body. Initially these neurites are indistinguishable, but ultimately one becomes the axon, whereas the others are converted to dendrites. The axon and dendrites then act as separate compartments, each associated with a unique set of motors and cargoes. More kinesin-1 is found in the developing axon compared with dendrites; this is likely induced by increased posttranslational modifications of axonal microtubules and the preference of kinesin-1 binding to these modified tracks (Jacobson et al. 2006; Hammond et al. 2010).

Many of the myriad nonmotor MAPs that bind microtubules (Hughes et al. 2008) are beginning to be recognized for their ability to influence motor activity and cargo transport. For example, tau, a widely studied neuronal MAP, decreases the frequency at which kinesin- 1 motors bind to microtubules (Seitz et al. 2002) and reduces the distance microtubule-bound kinesin-1 travels in in vitro experiments (Dixit et al. 2008). These findings on tau led to the longstanding assumption that MAPs inhibit microtubulebased transport either by saturating motor-binding sites along the microtubule or acting as "roadblocks" for motors (Baas and Qiang 2005; Leduc et al. 2012). Indeed, two additional MAPs, protein kinase Darkener of apricot (DOA) and its substrate, translational elongation factor EF1 $\gamma$, inhibit the transport of multiple classes of organelles in both directions along microtubules (Serpinskaya et al. 2013).

However, not all MAPs inhibit transport on microtubules. For example, ensconsin/MAP7 recruits kinesin-1 to microtubules (Sung et al. 2008) and is required for subsequent cargo transport (Barlan et al. 2013a). Interestingly, ensconsin does not require its microtubule-binding ability to promote cargo transport, although its binding to microtubules is likely important for preventing nonproductive activation in the absence of microtubule tracks (Barlan et al. 2013a).

Combinations of various tubulin isoforms, posttranslational modifications, and MAPs create a variety of microtubules with different affinities for molecular motors. These subpopulations of microtubules might act as separate compartments for the transport of specific cargoes. Restricting particular classes of cargo to a microtubule subpopulation increases the likelihood that the cargoes could interact and communicate, providing further order throughout the cytoplasm. Although cytoskeletal filaments primarily function as the tracks along which cellular cargoes are transported, we will also discuss below another important function for cytoskeletal filaments in retaining the cargoes at their destinations once they have been delivered.

\subsection{Motor-Track Interactions Can Influence Cargo Transport Indirectly}

In addition to specific, directed transport, motors can also influence cargo distribution indirectly through rearrangement of cytoskeletal tracks. Both kinesin-1 and dynein can slide microtubules against each other or the actin cortex (Jolly et al. 2010; Lu et al. 2013; Tanenbaum et al. 2013; del Castillo et al. 2015). This behavior is important to build the long, polarized axon during neurite outgrowth ( $\mathrm{Lu}$ et al. 2013; del Castillo et al. 2015) and also to rebuild this structure following repairable injury (Lu et al. 2015). Organelles that are associated with a given microtubule can thus be moved indirectly as a result of the motor-dependent movements of the track itself (Kulic et al. 2008).

Active cytoskeletal rearrangements by motors also contribute to diffusional movements of organelles throughout the cytoplasm. Inhibition of myosin II activity by treatment with the drug blebbistatin limits the cytoplasmic diffusion of organelle-sized beads (Guo et al. 2014). This suggests that random, active, motor-induced rearrangement of the actin cytoskeleton can contribute to the diffusive mobility of large particles throughout the cell. It is easy to imagine that fluctuations of cytoskeletal filaments are also important during directed, motor-dependent transport of large organelles and vesicles through the highly crowded cytoplasm.

Just as motor-cargo interactions are important for cargo transport and distribution, so are motor-track interactions. Furthermore, motor-track interactions can influence cargo distribution indirectly, even in the absence of specific motor-cargo associations. Each of these types of associations might be independently regulated by multiple inputs to allow for precise control over the rate and direction of transport, as well as the type of cargo.

\subsection{Regulatory Mechanisms Are Influenced by Cellular Context}

The degree to which cargo transport is regulated, and to some extent the mode of regulation used, is largely determined by the cellular context and physiological environment in which a transport event occurs. Observations of differences in cargo motility across cell types allow us to speculate on the biology behind the mechanisms that control these processes.

In a cell in the steady state, in which the main objective is homeostasis, organelles are shuttled back and forth to 
maintain a distribution characteristic of each organelle class. A change in net displacement is likely determined by the relative activity of multiple motors attached to each organelle type. Kinesins and dyneins work against one another, as in a tug-of-war, and a change in the direction of transport occurs when one motor gains an advantage in number over the other. In the absence of cellular and environmental signals, mechanisms regulating directional control are not engaged and very little net displacement of organelles is observed. In the case of stimulated transport, a physiological signal, such as a hormone or growth factor, induces cargo motility. This type of regulation is usually specific for a given cargo type and is often mediated through modification of the subset of motors associated with that specific cargo.

The strictest regulation of cargo transport is required in specialized, highly polarized cell types, such as embryos and neurons. The architecture of neurons, in particular, demands that motor-driven cargo delivery be efficient enough to span long distances into and out of the axon, while remaining flexible enough to undergo rapid changes during high levels of synaptic activity.

\section{EXAMPLES OF REGULATED TRANSPORT}

\subsection{Neuronal Maturation of Autophagosomes Requires Long-Range, Dynein-Driven Transport}

Autophagy is a mechanism through which cytoplasmic proteins, as well as organelles, can be degraded and their biochemical building blocks recycled. Cells use specific organelles, called autophagosomes, to act as the degradation machinery (Xie and Klionsky 2007). Two adaptor proteins, JIP1 and LC3, control the direction of autophagosome transport (Fu et al. 2014). LC3 localizes to the autophagosome during its biogenesis in the distal tip of the axon and recruits JIP1 to the organelle. JIP1 interacts with either plus end-directed kinesin or minus end-directed dynein/dynactin, but not both at the same time (Blasius et al. 2007; $\mathrm{Fu}$ and Holzbaur 2013). Site-specific phosphorylation of JIP1 regulates which motor JIP1 binds and thus acts as a molecular switch that determines the direction of cargo transport (Fu and Holzbaur 2013).

The switch-like behavior of JIP1 in this system is regulated by multiple inputs. Although JIP1 does not influence autophagosome motility in the distal axon tip, it is essential for uninterrupted, dynein-driven transport once the organelle enters into the distal axon. However, both in vitro and in vivo experiments have shown that interaction with JIP1 stimulates kinesin motility (Blasius et al. 2007; Fu and Holzbaur 2013), making its requirement for dynein-mediated movement seemingly paradoxical. On autophago- somes, JIP1-mediated activation of kinesin is inhibited by LC3. This allows dynein activity to predominate and power the organelles proximally down the axon. Furthermore, the phosphatase MKP1 colocalizes with JIP1 on autophagosomes and might function to keep JIP1 in its dephosphorylated state, which would further bias its association with dynein, rather than kinesin (Fu et al. 2014). During autophagosome transport, then, the motor-activating activity of JIP1 is influenced by multiple inputs specific to one class of cargo. The end result is a highly regulated mechanism of directed, long-range cargo transport down the axon.

This mode of transport is especially important for autophagosome function. In neurons, the direction of autophagosome transport is closely related to the maturation of the organelle. After their generation in the axon distal tip, autophagosomes undergo long-distance dynein-dependent transport, which is largely uninterrupted by kinesin-mediated motility events. As they move toward the cell body, autophagosomes interact and fuse with endo/lysosomal compartments, completing their maturation into fully acidified compartments. Only when autophagosomes are completely mature do they again display bidirectional and/or plus end-directed motility (Maday et al. 2012; Maday and Holzbaur 2014). Because these interorganelle interactions do not occur in the distal tip, translocation toward the cell body appears to be required for autophagosome maturation and the ultimate degradation of their contents (Maday et al. 2012).

\subsection{Numerous Physiological Stimuli Regulate Mitochondrial Motility}

Axonal mitochondrial transport provides a beautiful example of how multiple physiological stimuli influence organelle movement through distinct mechanisms (Fig. 2). Two proteins, the mitochondrial receptor Miro and its interaction partner Milton, an adaptor protein, recruit kinesin to mitochondria (Glater et al. 2006). Milton makes mitochondrial transport sensitive to glucose, whereas Miro transduces responses to intracellular $\mathrm{Ca}^{2+}$ levels.

Mitochondrial motility in axons is sensitive to glucose levels through the activity of the enzyme O-GlcNAc transferase (OGT). OGT, whose activity depends on glucose availability, is recruited to mitochondria by Milton. OGT catalyzes the addition of moiety-acetyl glucosamine to residues of numerous cellular substrates, including Milton; this process is called O-GlcNAcylation (OGA). When the glucose concentration is high, OGT mediates OGA of Milton, which results in the arrest of mitochondrial motility (Fig. 2) (Pekkurnaz et al. 2014). Thus Milton is an example of an adaptor protein that recruits both a motor and regulatory factor to the organelle. Although the mechanism 
A

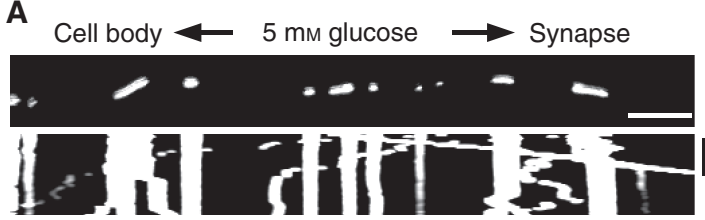

$30 \mathrm{~mm}$ glucose

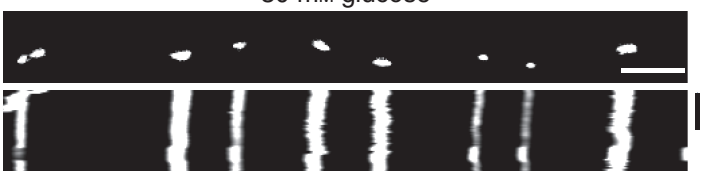

B

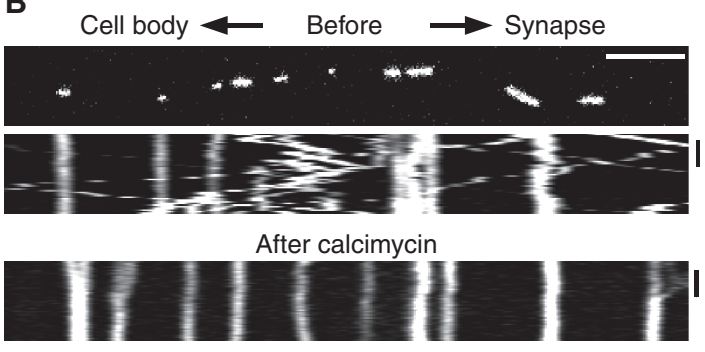

C


Figure 2. Multiple physiological inputs regulate the transport of mitochondria in neurons. $(A, B)$ Kymographs showing mitochondria moving in axons of mouse hippocampal neurons. The $y$-axis of each kymograph represents time, and the $x$-axis depicts the position of the organelles such that stationary organelles appear as vertical lines, whereas moving organelles are diagonals. The first frame of a time-lapse movie is shown above each kymograph. Scale bars in kymographs are $10 \mu \mathrm{m}$ and $100 \mathrm{sec}$. Mitochondria motility is arrested in response to $(A)$ increased glucose, as a result of the activity of $O$-GlcNAc transferase (OGT) on the kinesin-binding protein Milton, and $(B)$ treatment with the ion-carrier antibiotic calcimycin, which increases intracellular $\mathrm{Ca}^{2+}$ levels. $(C)$ Cartoon showing how $\mathrm{Ca}^{2+}$ inhibits the transport of mitochondria. When $\mathrm{Ca}^{2+}$ is bound to the mitochondrial protein Miro, the interaction of Miro with the kinesin motor domain prevents kinesin from binding to the microtubule. $(D)$ Schematic showing some of the numerous factors regulating mitochondrial motility in neurons, including the E3 ubiquitin ligase Parkin, the serine/threonine-protein kinase PINK1, OGT, the receptor Miro and its partner Milton, and the mitochondria-associated protein syntaphilin. (Images courtesy of Gulcin Pekkurnaz and Tom Schwarz; a portion of the figure was modified from Wang and Schwarz 2009, with permission from Elsevier.) 
through which Milton OGA affects transport is not yet known, Milton allows OGT to function as a sort of mitochondrial glucose sensor and might help mitochondria "park" in glucose-rich regions to promote efficient ATP synthesis using glucose as a carbon source.

Miro regulates mitochondrial motility in response to intracellular $\mathrm{Ca}^{2+}$ by controlling the association of kinesin with the microtubule. At physiological $\mathrm{Ca}^{2+}$ levels, Miro interacts with the tail of kinesin; this interaction strictly depends on Milton. At high $\mathrm{Ca}^{2+}$ levels, $\mathrm{Ca}^{2+}$ binding to the two EF-hand domains of Miro induces a conformational change that enables Miro to interact with the motor domains of kinesin independently of Milton (Fig. 2C). This interaction prevents kinesin from binding to microtubules and results in the arrest of mitochondria movement (Wang and Schwarz 2009). Similar to the glucose example above, this sensitivity to $\mathrm{Ca}^{2+}$ might allow mitochondria to "park" in regions with low ATP concentrations, in which $\mathrm{Ca}^{2+}$-transport mechanisms falter and cytoplasmic $\mathrm{Ca}^{2+}$ accumulates. Importantly, even in areas of elevated $\mathrm{Ca}^{2+}$, kinesin remains bound to the mitochondria through its cargo-binding association with Milton, enabling transport of the organelle to resume immediately once $\mathrm{Ca}^{2+}$ levels return to normal.

In a second, distinct regulatory mechanism, Miro completely dissociates from mitochondria, disrupting the entire kinesin-adaptor complex. The mechanism involves two proteins-PINK1 and Parkin - whose mutations have been linked to Parkinson's disease. Together, these proteins target Miro for degradation in response to mitochondrial damage. The PINK1 serine/threonine-protein kinase phosphorylates Miro; this modification allows Parkin, an E3 ubiquitin ligase, to designate Miro for removal from the mitochondrial membrane and destruction in the proteasome. Mitochondria-lacking Miro cannot bind kinesin, and so their motility stops. Thus, in normally functioning neurons, the PINK1 - Parkin pathway prevents kinesin-mediated transport of damaged mitochondria, possibly as a way of conserving resources and marking the organelles for degradation (Wang et al. 2011).

Mitochondrial biology also relies on a population of stationary organelles to perform chemical reactions in particular locations within the neuron. Insight into the ways in which mitochondria are anchored along the axon has come from studies of the mitochondria-associated protein syntaphilin. Syntaphilin appears to immobilize mitochondria in two ways. First, it uses a microtubule-binding domain to tether the organelle to the cytoskeletal track and prevent its movement (Kang et al. 2008). Second, syntaphilin can bind kinesin directly and appears to compete with Milton for motor association, ultimately leading to the detachment of kinesin from mitochondria (Chen and Sheng
2013). Whether they are and how the associations of syntaphilin with microtubules and kinesin are coordinated or regulated remains to be determined. Nonetheless, immobilization of mitochondria in the axon has clear implications for neuronal physiology. Demyelinated axons, such as those in patients with multiple sclerosis, appear particularly sensitive to the distribution of mitochondria. When demyelinated, axons contain more stationary mitochondria than normal; this effect is dependent on syntaphilin. These stationary mitochondria might provide a protective effect, as demyelinated axons degenerate faster in the absence of syntaphilin-mediated mitochondria tethering (Ohno et al. 2014).

\subsection{Melanosome Distribution Requires Distinct Roles for Both Microtubules and Actin}

The distribution of pigment granules in cells called melanocytes elegantly shows how the distinct arrangements of microtubules and actin filaments allow for complementary forms of cargo transport and delivery. Melanocytes synthesize and maintain melanin pigment in dedicated organelles called melanosomes. After synthesis, melanosomes undergo fast transport along microtubules into the distal processes (called dendrites) of melanocytes. There, a tripartite complex comprising myosin-Va, the small GTPase Rab27a, and the adaptor protein melanophilin anchors them to actin filaments in the cell periphery (Hume and Seabra 2011). The tethering of melanosomes in melanocyte dendrites is essential for their function; ultimately, melanosomes are transferred from dendrites to neighboring keratinocytes, where they provide visible pigmentation in the skin and offer protection from damaging ultraviolet light. Disruption of the tethering complex results in a loss of melanosomes from dendrites (Fig. 3) (Wu et al. 1997, 2002) and, consequently, a lack of pigment transfer into keratinocytes. Indeed, the importance of organelle tethering in melanocytes was first discovered through examination of coat-color mutants in mice. Disrupted melanosome transfer produces a lightening effect in the coats of mutants, which led to the naming of the mutant genes dilute (myosin-Va), ashen (Rab27a), and leaden (melanophilin) (Fig. 3). In humans, mutations in these proteins can lead to ocular and/or cutaneous albinism, in addition to other neurological disorders.

In contrast to mammalian systems, hormones acutely regulate melanosome transport in lower vertebrates (fish and amphibians). In response to hormone secretion, melanosomes are transported synchronously toward or away from the cell center, which changes the pigment distribution in individual cells. Cyclic AMP (cAMP)-dependent 



Figure 3. Obtaining a uniform distribution of melanosomes in cultured melanocytes requires a tripartite actintethering complex. The two rows are matched bright-field $(A-D)$ images showing pigmented melanosomes, and phase-contrast $(E-H)$ images showing the shapes of the cells. Melanocytes are from $(A, E)$ wild-type mice, $(B, F)$ ashen mice with a mutation in the small GTPase Rab27a, $(C, G)$ leaden mice with a mutation of the adaptor protein melanophilin, and $(D, H)$ dilute mice with a mutation of the motor myosin-Va. Melanosomes are uniformly distributed in melanocytes from wild-type mice, but are not anchored to actin filaments in the cell periphery of melanocytes from the mutant mice. This change in pigment distribution compromises melanosome transfer from melanocytes to keratinocytes and results in a lightening of the coat colors of mutant mice. Bar, $12 \mu \mathrm{m}$. (Images courtesy of Xufeng Wu and John Hammer. This work originally appeared in Wu et al. 2002; G, reprinted, with permission from Macmillan Publishers Ltd., from Wu et al. 2002.)

protein kinase (PKA) is the key upstream regulator of melanosome distribution in these cells; the effectors downstream of PKA are not known (Reilein et al. 1998; Sheets et al. 2007). The hormones melatonin and epinephrine induce a decrease in cytoplasmic cAMP concentration, which reduces PKA activity. This causes dynein motors to aggregate melanosomes toward the cell center. Conversely, melanocyte-stimulating hormone increases cAMP concentrations and PKA activity, which promotes kinesin-2-mediated dispersion of the pigment organelles throughout the cell. As in mammalian cells, the actin-dependent tethering complex complements the microtubule cytoskeleton and is required to maintain a uniform melanosome distribution throughout the cell.

Melanosome distribution can be further fine-tuned by other regulatory modes that work in parallel to hormone secretion. For example, in Xenopus melanophores, the ubiquitous microtubule-binding protein MAP4 inhibits dynein activity. However, site-specific phosphorylation of MAP4 reduces its ability to bind microtubules and inhibit dynein activity. Aggregation-inducing signals increase
MAP4-specific phosphorylation, releasing MAP4 from microtubules, and allowing dynein to aggregate melanosomes toward the cell center (Semenova et al. 2014).

\subsection{Protein Kinase A-Dependent Cargo Switching}

Although the molecular details underlying PKA-mediated regulation of melanosomes are not well understood, sitespecific PKA-dependent phosphorylation has been shown to mediate a cargo-switching phenomenon during viral infection (Scherer et al. 2014). Viruses use cytoskeletal transport mechanisms to reach their replication sites and as a route for newly assembled viral particles to exit the infected cell (Greber and Way 2006). The motility of viruses is controlled in much the same way as host cellular cargoes.

During adenovirus infection, PKA phosphorylates dynein light intermediate chain 1 (LIC1), one of the cargobinding subunits of dynein. This modification to LIC1 has two reciprocal effects. Phosphorylated LIC1 mediates the 
interaction between dynein and adenovirus by binding to the viral capsid protein hexon. This interaction promotes viral transport toward the nucleus. LIC1 phosphorylation also dissociates LIC1 from the lysosomal adaptor protein RILP (Rab7-interacting lysosomal protein). This release from dynein causes lysosomes, which normally cluster near the nucleus, to disperse outward toward the cell periphery. The acute transport of these degradative organelles might show a specific effort by the host cell to combat the effects of infection (Scherer et al. 2014). Additional studies are needed to determine how widespread PKA-dependent cargo switching is and what other types of interactions PKA-mediated phosphorylation influences. Interestingly, melanosomes are a special derivative of lysosomes. It would be interesting to see whether dynein LIC phosphorylation also regulates melanosome transport.

\section{CONCLUSION}

Motor proteins and their cytoskeletal tracks are essential for organizing membrane compartments and other components within a cell. Multiple molecular mechanisms regulate both tracks and motors, and it is likely that many more examples of regulation over individual motors will be uncovered. Some of these mechanisms include multiple levels of regulation and more than one input. The studies detailed here show how changes in transport can result from specific changes in the cellular environment and illustrate that modifying one component of a transport pathway often has multiple downstream effects. There is mounting evidence to suggest that specific pathways regulate each motor-cargo combination independently, and that these regulatory pathways can be further fine-tuned for specific types of cells and, in particular, physiological situations. Through the use of multiple layers of control, the distribution and trafficking of individual membrane compartments can be precisely honed to allow the cell to adapt to ever-changing environments.

\section{ACKNOWLEDGMENTS}

We are extremely grateful to Nico Camargo for creating the illustrations used in Figure 1. We also extend sincere thanks to Gulcin Pekkurnaz and Tom Schwarz for providing illustrations and micrographs used in Figure 2, as well as Xufeng Wu and John Hammer for micrographs used in Figure 3. Research in the Gelfand laboratory is supported by the National Institute of General Medical Science of the National Institutes of Health under award numbers R01GM052111 and P01GM096971. K.B. is a Howard Hughes Medical Institute Fellow of the Life Sciences Research Foundation.

\section{REFERENCES}

* Reference is also in this subject collection.

Akhmanova A, Hammer JA III. 2010. Linking molecular motors to membrane cargo. Curr Opin Cell Biol 22: 479-487.

Baas PW, Qiang L. 2005. Neuronal microtubules: When the MAP is the roadblock. Trends Cell Biol 15: 183-187.

Barlan K, Gelfand VI. 2010. Intracellular transport: ER and mitochondria meet and greet along designated tracks. Curr Biol 20: R845-R847.

Barlan K, Lu W, Gelfand VI. 2013a. The microtubule-binding protein ensconsin is an essential cofactor of kinesin-1. Curr Biol 23: 317-322.

Barlan K, Rossow MJ, Gelfand VI. 2013b. The journey of the organelle: Teamwork and regulation in intracellular transport. Curr Opin Cell Biol 25: 483-488.

Blasius TL, Cai D, Jih GT, Toret CP, Verhey KJ. 2007. Two binding partners cooperate to activate the molecular motor Kinesin-1. J Cell Biol 176: $11-17$.

Bowman AB, Kamal A, Ritchings BW, Philp AV, McGrail M, Gindhart JG, Goldstein LS. 2000. Kinesin-dependent axonal transport is mediated by the sunday driver (SYD) protein. Cell 103: 583-594.

Cai D, McEwen DP, Martens JR, Meyhofer E, Verhey KJ. 2009. Single molecule imaging reveals differences in microtubule track selection between Kinesin motors. PLoS Biol 7: e1000216.

Chen Y, Sheng ZH. 2013. Kinesin-1-syntaphilin coupling mediates activity-dependent regulation of axonal mitochondrial transport. J Cell Biol 202: $351-364$.

Chevalier-Larsen E, Holzbaur ELF. 2006. Axonal transport and neurodegenerative disease. Biochimica Biophys Acta 1762: 1094-1108.

del Castillo U, Winding M, Lu W, Gelfand VI. 2015. Interplay between kinesin-1 and cortical dynein during axonal outgrowth and microtubule organization in Drosophila neurons. eLife 4: e10140.

Dixit R, Ross JL, Goldman YE, Holzbaur ELF. 2008. Differential regulation of dynein and kinesin motor proteins by $\tau$. Science 319: 10861089.

FriedmanJR, Webster BM, Mastronarde DN, Verhey KJ, Voeltz GK. 2010. ER sliding dynamics and ER-mitochondrial contacts occur on acetylated microtubules. J Cell Biol 190: 363-375.

Friedman JR, Lackner LL, West M, DiBenedetto JR, Nunnari J, Voeltz GK. 2011. ER tubules mark sites of mitochondrial division. Science 334: $358-362$.

Fu MM, Holzbaur ELF. 2013. JIP1 regulates the directionality of APP axonal transport by coordinating kinesin and dynein motors. $J$ Cell Biol 202: 495-508.

Fu M-M, Holzbaur ELF. 2014. Integrated regulation of motor-driven organelle transport by scaffolding proteins. Trends Cell Biol 24: $564-$ 574.

Fu M-M, Nirschl JJ, Holzbaur ELF. 2014. LC3 binding to the scaffolding protein JIP1 regulates processive dynein-driven transport of autophagosomes. Dev Cell 29: 577-590.

Gindhart JG, Chen J, Faulkner M, Gandhi R, Doerner K, Wisniewski T, Nandlestadt A. 2003. The kinesin-associated protein UNC-76 is required for axonal transport in the Drosophila nervous system. Mol Biol Cell 14: 3356-3365.

Glater EE, Megeath LJ, Stowers RS, Schwarz TL. 2006. Axonal transport of mitochondria requires milton to recruit kinesin heavy chain and is light chain independent. J Cell Biol 173: 545-557.

* Goodson HV, Jonasson EM. 2016. Microtubules and microtubuleassociated proteins. Cold Spring Harb Perspect Biol doi: 101101/ cshperspect.a022608.

Greber UF, Way M. 2006. A superhighway to virus infection. Cell 124: $741-754$.

Guo M, Ehrlicher AJ, Jensen MH, Renz M, Moore JR, Goldman RD, Lippincott-Schwartz J, Mackintosh FC, Weitz DA. 2014. Probing the 
stochastic, motor-driven properties of the cytoplasm using force spectrum microscopy. Cell 158: 822-832.

Hammond JW, Huang C-F, Kaech S, Jacobson C, Banker G, Verhey KJ. 2010. Posttranslational modifications of tubulin and the polarized transport of kinesin-1 in neurons. Mol Biol Cell 21: 572583.

Hirokawa N, Niwa S, Tanaka Y. 2010. Molecular motors in neurons: Transport mechanisms and roles in brain function, development, and disease. Neuron 68: 610-638.

Hughes JR, Meireles AM, Fisher KH, Garcia A, Antrobus PR, Wainman A, Zitzmann N, Deane C, Ohkura H, Wakefield JG. 2008. A microtubule interactome: Complexes with roles in cell cycle and mitosis. PLoS Biol 6: e98.

Hume AN, Seabra MC. 2011. Melanosomes on the move: A model to understand organelle dynamics. Biochem Soc Trans 39: $1191-1196$.

Jacobson C, Schnapp B, Banker GA. 2006. A change in the selective translocation of the Kinesin-1 motor domain marks the initial specification of the axon. Neuron 49: 797-804.

Janke C. 2014. The tubulin code: Molecular components, readout mechanisms, and functions. J Cell Biol 206: 461-472.

Jolly AL, Kim J, Srinivasan D, Lakonishok M, Larson AG, Gelfand VI. 2010. Kinesin-1 heavy chain mediates microtubule sliding to drive changes in cell shape. Proc Natl Acad Sci 107: 12151-12156.

Kang J-S, Tian J-H, Pan P-Y, Zald P, Li C, Deng C, Sheng Z-H. 2008. Docking of axonal mitochondria by syntaphilin controls their mobility and affects short-term facilitation. Cell 132: 137-148.

Konishi Y, Setou M. 2009. Tubulin tyrosination navigates the kinesin-1 motor domain to axons. Nat Neurosci 12: 559-567.

Kulic IM, Brown AEX, Kim H, Kural C, Blehm B, Selvin PR, Nelson PC, Gelfand VI. 2008. The role of microtubule movement in bidirectional organelle transport. Proc Natl Acad Sci 105: 10011-10016.

Leduc C, Padberg-Gehle K, Varga V, Helbing D, Diez S, Howard J. 2012. Molecular crowding creates traffic jams of kinesin motors on microtubules. Proc Natl Acad Sci 109: 6100-6105.

Leite F, Way M. 2015. The role of signalling and the cytoskeleton during Vaccinia Virus egress. Virus Res 209: 87-99.

Liao G, Gundersen GG. 1998. Kinesin is a candidate for cross-bridging microtubules and intermediate filaments. Selective binding of kinesin to detyrosinated tubulin and vimentin. J Biol Chem 273: 9797-9803.

Lu W, Fox P, Lakonishok M, Davidson MW, Gelfand VI. 2013. Initial neurite outgrowth in Drosophila neurons is driven by kinesin-powered microtubule sliding. Curr Biol 23: 1018-1023.

Lu W, Lakonishok M, Gelfand VI. 2015. Kinesin-1-powered microtubule sliding initiates axonal regeneration in Drosophila cultured neurons. Mol Biol Cell 26: 1296-1307.

Maday S, Holzbaur ELF. 2014. Autophagosome biogenesis in primary neurons follows an ordered and spatially regulated pathway. Dev Cell 30: $71-85$.

Maday S, Wallace KE, Holzbaur ELF. 2012. Autophagosomes initiate distally and mature during transport toward the cell soma in primary neurons. J Cell Biol 196: 407-417.

Matanis T, Akhmanova A, Wulf P, Del Nery E, Weide T, Stepanova T, Galjart N, Grosveld F, Goud B, de Zeeuw CI, et al. 2002. Bicaudal-D regulates COPI-independent Golgi-ER transport by recruiting the dynein-dynactin motor complex. Nat Cell Biol 4: 986-992.

McKenney RJ, Huynh W, Tanenbaum ME, Bhabha G, Vale RD. 2014. Activation of cytoplasmic dynein motility by dynactin-cargo adapter complexes. Science 345: 337-341.

Morikawa M, Yajima H, Nitta R, Inoue S, Ogura T, Sato C, Hirokawa N. 2015. X-ray and Cryo-EM structures reveal mutual conformational changes of Kinesin and GTP-state microtubules upon binding. EMBO J 34: 1270-1286.
Ohno N, Chiang H, Mahad DJ, Kidd GJ, Liu L, Ransohoff RM, Sheng ZH, Komuro H, Trapp BD. 2014. Mitochondrial immobilization mediated by syntaphilin facilitates survival of demyelinated axons. Proc Natl Acad Sci 111: 9953-9958.

Pekkurnaz G, Trinidad JC, Wang X, Kong D, Schwarz TL. 2014. Glucose regulates mitochondrial motility via Milton modification by $\mathrm{O}$ GlcNAc transferase. Cell 158: 54-68.

Reed NA, Cai D, Blasius TL, Jih GT, Meyhofer E, Gaertig J, Verhey KJ. 2006. Microtubule acetylation promotes kinesin-1 binding and transport. Curr Biol 16: 2166-2172.

Reilein AR, Tint IS, Peunova NI, Enikolopov GN, Gelfand VI. 1998. Regulation of organelle movement in melanophores by protein kinase A (PKA), protein kinase $\mathrm{C}$ (PKC), and protein phosphatase $2 \mathrm{~A}$ (PP2A). J Cell Biol 142: 803-813.

Scherer J, Yi J, Vallee RB. 2014. PKA-dependent dynein switching from lysosomes to adenovirus: A novel form of host-virus competition. $J$ Cell Biol 205: 163-177.

Seitz A, Kojima H, Oiwa K, Mandelkow E-M, Song Y-H, Mandelkow E. 2002. Single-molecule investigation of the interference between kinesin, $\tau$ and MAP2c. EMBO J 21: 4896-4905.

Sellers JR, Thirumurugan K, Sakamoto T, Hammer JA, Knight PJ. 2008. Calcium and cargoes as regulators of myosin $5 \mathrm{a}$ activity. Biochem Biophys Res Commun 369: 176-181.

Semenova I, Ikeda K, Resaul K, Kraikivski P, Aguiar M, Gygi S, Zaliapin I, Cowan A, Rodionov V. 2014. Regulation of microtubule-based transport by MAP4. Mol Biol Cell 25: 3119-3132.

Serpinskaya AS, Tuphile K, Rabinow L, Gelfand VI. 2013. Protein kinase Darkener of apricot and its substrate EF1 $\gamma$ regulate organelle transport along microtubules. J Cell Sci 127: 33-39.

Sheets L, Ransom D, Mellgren E, Johnson S, Schnapp B. 2007. Zebrafish melanophilin facilitates melanosome dispersion by regulating dynein. Curr Biol 17: 1721-1734.

Sirajuddin M, Rice LM, Vale RD. 2014. Regulation of microtubule motors by tubulin isotypes and post-translational modifications. Nat Cell Biol 16: 335-344.

Smith DS, Niethammer M, Ayala R, Zhou Y, Gambello MJ, WynshawBoris A, Tsai LH. 2000. Regulation of cytoplasmic dynein behaviour and microtubule organization by mammalian Lis1. Nat Cell Biol 2: 767-775.

Stowers RS, Megeath LJ, Górska-Andrzejak J, Meinertzhagen IA, Schwarz TL. 2002. Axonal transport of mitochondria to synapses depends on Milton, a novel Drosophila protein. Neuron 36: 10631077.

Sung H-H, Telley IA, Papadaki P, Ephrussi A, Surrey T, Rørth P. 2008. Drosophila ensconsin promotes productive recruitment of Kinesin-1 to microtubules. Dev Cell 15: 866-876.

* Svitkina T. 2016. The actin cytoskeleton and actin-based motility. Cold Spring Harb Perspect Biol doi: 101101/cshperspect.a018267.

* Sweeney HL, Holzbaur ELF. 2016. Motor proteins. Cold Spring Harb Perspect Biol doi: 101101/cshperspect.a021931.

Szyk A, Deaconescu AM, Spector J, Goodman B, Valenstein ML, Ziolkowska NE, Kormendi V, Grigorieff N, Roll-Mecak A. 2014. Molecular basis for age-dependent microtubule acetylation by tubulin acetyltransferase. Cell 157: 1405-1415.

Tanenbaum ME, Vale RD, McKenney RJ. 2013. Cytoplasmic dynein crosslinks and slides anti-parallel microtubules using its two motor domains. eLife 2: $\mathrm{e} 00943$.

Tischfield MA, Engle EC. 2010. Distinct $\alpha$ - and $\beta$-tubulin isotypes are required for the positioning, differentiation and survival of neurons: New support for the 'multi-tubulin' hypothesis. Biosci Rep 30: 319330.

Verhey KJ, Hammond JW. 2009. Traffic control: Regulation of kinesin motors. Nat Rev Mol Cell Biol 10: 765-777.

Verhey KJ, Kaul N, Soppina V. 2011. Kinesin assembly and movement in cells. Annu Rev Biophys 40: 267-288. 
Wang X, Schwarz TL. 2009. The mechanism of $\mathrm{Ca}^{+}$-dependent regulation of kinesin-mediated mitochondrial motility. Cell 136: $163-174$

Wang X, Winter D, Ashrafi G, Schlehe J, Wong YL, Selkoe D, Rice S, Steen J, LaVoie MJ, Schwarz TL. 2011. PINK1 and Parkin target Miro for phosphorylation and degradation to arrest mitochondrial motility. Cell 147: 893-906.

Westermann S, Weber K. 2003. Post-translational modifications regulate microtubule function. Nat Rev Mol Cell Biol 4: 938947.
Wu X, Bowers B, Wei Q, Kocher B, Hammer JA. 1997. Myosin Vassociates with melanosomes in mouse melanocytes: Evidence that myosin $\mathrm{V}$ is an organelle motor. J Cell Sci 110: 847-859.

Wu XS, Rao K, Zhang H, Wang F, Sellers JR, Matesic LE, Copeland NG, Jenkins NA, Hammer JA. 2002. Identification of an organelle receptor for myosin-Va. Nat Cell Biol 4: 271-278.

Xie Z, Klionsky DJ. 2007. Autophagosome formation: Core machinery and adaptations. Nat Cell Biol 9: 1102-1109.

Yadav S, Puthenveedu MA, Linstedt AD. 2012. Golgin160 recruits the dynein motor to position the Golgi apparatus. Dev Cell 23: 153-165. 




\title{
Microtubule-Based Transport and the Distribution, Tethering, and Organization of Organelles
}

\author{
Kari Barlan and Vladimir I. Gelfand \\ Cold Spring Harb Perspect Biol 2017; doi: 10.1101/cshperspect.a025817
}

\section{Subject Collection The Cytoskeleton}

Microtubules and Microtubule-Associated

Proteins

Holly V. Goodson and Erin M. Jonasson

Motor Proteins

H. Lee Sweeney and Erika L.F. Holzbaur

Myosin-Driven Intracellular Transport Margaret $A$. Titus

The Actin Cytoskeleton and Actin-Based Motility Tatyana Svitkina

Mechanical Properties of the Cytoskeleton and Cells

Adrian F. Pegoraro, Paul Janmey and David A. Weitz

Intermediate Filaments and the Regulation of Cell Motility during Regeneration and Wound Healing Fang Cheng and John E. Eriksson

Intermediate Filaments and the Plasma Membrane Jonathan C.R. Jones, Chen Yuan Kam, Robert M. Harmon, et al.

\section{Overview of the Cytoskeleton from an \\ Evolutionary Perspective}

Thomas D. Pollard and Robert D. Goldman

Types I and II Keratin Intermediate Filaments Justin T. Jacob, Pierre A. Coulombe, Raymond Kwan, et al.

Muscle Contraction H. Lee Sweeney and David W. Hammers

Type III Intermediate Filaments Desmin, Glial Fibrillary Acidic Protein (GFAP), Vimentin, and Peripherin Elly M. Hol and Yassemi Capetanaki

Cytokinesis in Metazoa and Fungi Michael Glotzer

Ciliary Motility: Regulation of Axonemal Dynein Motors

Rasagnya Viswanadha, Winfield S. Sale and Mary E. Porter

Actin-Based Adhesion Modules Mediate Cell Interactions with the Extracellular Matrix and Neighboring Cells Alexia I. Bachir, Alan Rick Horwitz, W. James Nelson, et al.

For additional articles in this collection, see http://cshperspectives.cshlp.org/cgi/collection/




Intracellular Motility of Intermediate Filaments Rudolf E. Leube, Marcin Moch and Reinhard Windoffer
Microtubule-Based Transport and the Distribution, Tethering, and Organization of Organelles Kari Barlan and Vladimir I. Gelfand

For additional articles in this collection, see http://cshperspectives.cshlp.org/cgi/collection/

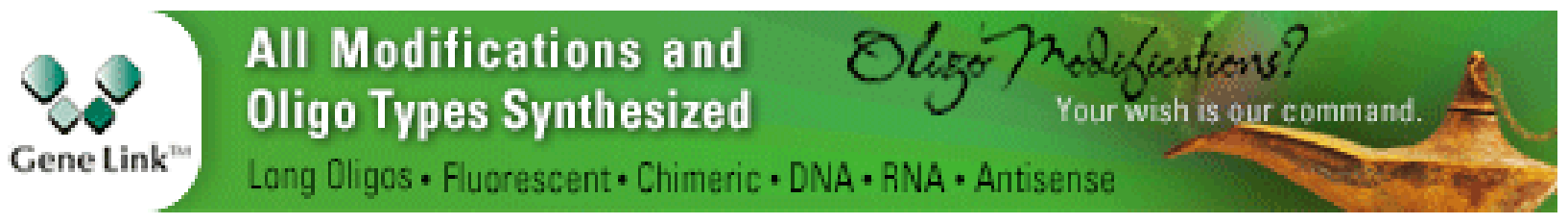

Copyright @ 2017 Cold Spring Harbor Laboratory Press; all rights reserved 